\title{
LAS PALABRAS ESPAÑOLAS CONTENIDAS EN EL VOCABULARIO DE LAS INTRODUCTIONES LATINAE DE ANTONIO DE NEBRIJA
}

\author{
ANTONIO QUILIS
}

Nebrija publicó en Salamanca, en 1481, sus Introductiones latinae ${ }^{1}$. Como es sabido, es una obra de inspiración pedagógica para la enseñanza del latín a los jóvenes, y su autor consiguió, entre otras cosas, que fuese concisa, clara y útil.

En las últimas páginas, incorporó un pequeño vocabulario (fol 49 v.s.n. hasta el final), que incluye las palabras que pueden tener dificultad para el estudiante. Este vocabulario ${ }^{2}$ tiene 955 entradas en latín, de las que 176 dan el significado o los significados en español; el número total de lexías españolas que aparecen asciende a 201: esto supone una frecuencia nada despreciable del $21 \%$ de palabras españolas.

En general, las definiciones de este vocabulario siguen las líneas del $\mathrm{DLE}^{3}$; por ejemplo: Calendae sunt primi dies mensium, Adoleo cresco uel incendo, Decas quod diuiditur per decem, Armenia est regno Asiae; otras veces, declara que no conoce el término, como en Maguderis herba est mihi incognita, o, simplemente, no hay ninguna aclaración, como en Argutiae, Cleo, Popisma.

Los lemas que contienen palabras españolas son, como es habitual, breves, y responden a la siguiente estructura:

\footnotetext{
${ }^{1}$ Reproducida facsimilarmente por la Universidad de Salamanca, con presentación de Pedro Amat y proemio de Eugenio de Bustos, en 1981.

${ }^{2}$ Según Adriana della Casa —en su trabajo «Le Introductiones latinae de Nebrija e il Catholicon di Giovanni Balbi», en Antonio de Nebrija: Edad Media y Renacimiento, ed. de Carmen Codoñer y Juan Antonio González Iglesias, Salamanca, Ediciones Universidad, 1994, págs. 237-245-, Nebrija tuvo presente para su elaboración el Catholicon de Giovanni Balbi

${ }^{3}$ Elio Antonio de Nebrija, Diccionario latino-español, Salamanca, 1492, edición facsimilar de Puvill-Editor, con estudio preliminar por Germán Colón y Amadeu-J. Soberanas, Barcelona, 1979.
}

$R F E$, LXXX, 2000, $1 .^{\circ}-2 .^{\circ}$, págs. 181-191 
a) «Entrada + est + Significado español»: Abigere est oxear o arredrar, Follis est la bala o el fuelle, Fornix est arcus o bóveda, Furfures sunt in capite la caspa, Glans est fructus silvestris alias bellota, Lis est contentio el pleyto o lid, Carex herba est el carrizo;

b) «Entrada + (est) + vulgo + (dicitur)»: Adamas vulgo el diamante, Aes aeris vulgo el cobre, Alumen est quod vulgo alumbre, Colus est vulgo la rueca, Crates vulgo dicitur el zarzo, Exoletus est qui vulgo dicitur paje;

c) «Entrada + Significado o significados españoles»: Ala et axilla el sobaco, Caenaculum el soberado, Carduelis auicola el sirguerito, Chorus ventus gallego, Dorcas ex genere cervino corza, Cucumis pepino o melon o cohombro, Cuculus el cuclillo o la cugulla o capiro, Lacus el lago o lagar o viga, y, más adelante, Lacunar est viga, es decir, 'hueco de un techo artesonado' $^{4}$, etc. En alguna ocasión, se extiende en explicaciones: Calix per $i$ iota el vaso, at vero per y psilon el erizo de la castaña, Coclear la cuchara in similitudinem cocleae, etc.

1. Pero, además, lo importante para nosotros de este vocabulario es que contiene 42 palabras españolas que, por ahora, se pueden datar aquí por primera vez: albahaca, almirón, cicorea 'achicoria', ámbar, canasto, capiro, carmeso, carnicol, caspa, chanela, cohombro, cuchara, cuclillo, cugulla, dátil, diamante, dormidera, empeyne, faraute, gryfo, harnero, hastio, higado, hoja, hoyo, láudano, lirón, malva loca, melón, morecillo, ordeñar, oruga, oxear, paje, papada, pegujal, pértigo, rayda, recalcar, regalar, soberado, trinchante. Algunas no aparecen en el Diccionario latino-español, como almirón, láudano, malva loca; otras no figuran en el Vocabulario españollatino, como canasto, carmeso - pero sí carmesí- y (cosa) raída.

2. En lo que sigue, damos primero la entrada por la palabra española; a continuación, toda la papeleta conforme aparece en las Introductiones; por último, confirmamos o no su presencia en los dos grandes repertorios lexicográficos nebrisenses.

Abonado. Praes est el fiador abonado. VEL ${ }^{5}$. DLE: «por abonado en raízes». Acelga. Betaceus a beta el acelga. VEL. DLE.

Aceña. Pistrinum est el molino o aceña. VEL. DLE.

Adobe. Laterem ladrillo o adobe. VEL. DLE.

\footnotetext{
${ }^{4}$ Para Raimundo de Miguel y el Marqués de Morante - Nuevo Diccionario latino-español etimológico, Madrid, 1878-, lacunar [lakúnar] es «Lagunar, techumbre de madera tallada con vigas a trechos».

${ }^{5}$ Vocabulario español-latino por Elio Antonio de Nebrija. Madrid, Real Academia Española, Edición facsimilar, 1989.
} 
Agricultura. Georgica opus de agricultura. DLE: «Georgica. orum. por libros de agricultura».

Aguijón. Aculeus ${ }^{6}$ est vulgo aguijón. VEL. DLE: aculeus, con tres entradas: "por el caxquillo de la saeta», "por el aguijón del abeja», "por cualquier aguijón o punta».

Álamo. Populus est arbor el álamo. VEL. DLE: «por el álamo blanco».

Albahaca. Ocymum est quod dicunt albahaca. VEL: «Albahaca ierva conocida. basilicum.i.». DLE. Primera documentación en $I L$.

Albañar. Cloaca vulgo albañar. VEL. DLE.

Alcançar. Potior est alcançar lo deseado. VEL: «Alcançar lo desseado. nanciscor. eris». DLE.

Alférez. Signifer est qui vulgo alférez. VEL. DLE.

Alfiler. Acicula vulgo el alfiler. VEL: «Alfilel. acicula. aciculae.». DLE: «por el alfilel o aguja pequeña».

Alguirnandas. Serta que dicuntur alguirnandas. VEL: «Alguirnalda de flores. serta. orum». DLE: «por alguirnalda».

Alhaja de casa. Supellex est alhaja de casa. VEL. DLE: «por alhaja».

Aljófar. Margaris est quae margarita aljófar. VEL

Almirón. Intybus herba vulgo cicorea o almirón. VEL ${ }^{7}$. Primera documentación en $I L$.

Almizque. Muscus est almizque vulgo. DLE: «Muscus. i. por el moho del árbol».

Alumbre. Alumen est quod vulgo alumbre. VEL. DLE: «por el alumbre piedra conocida».

Alvayalde. Cerussa ${ }^{8}$ vulgo el aluayalde. VEL.

Ámbar. Helectrum quod vulgo ámbar. vel. Primera documentación en $I L$.

Amiento. Amentum quod vulgo amiento. VEL: «Amiento para tirar. amentum.i.». DLE.

Arayhán. Myrtus vulgo el arayhán. VEL: «Arraihan». DLE: «por el arraihán morisco».

Arbor. V. Hoja.

Arredrar. Abigere est oxear o arredrar. VEL. DLE: con dos entradas, con los significados de «hurtar ganados» y de «oxear o espantar aves».

Assador. Veru est quod vulgo assador. VEL: «Assadero para assar. veru.u. indecli.». DLE.

Asechança. Insidiae asechança o celada. VEL ${ }^{9}$. DLE.

\footnotetext{
6 En las Introductiones, acultus

7 «Intubus.i.».

${ }^{8}$ En las Introductiones, cerusa.

9 «Assechança».
} 
Astilla. Fomes est quae vulgo la astilla. vEL: «Astilla de madera. assula. ae.». DLE: «por la iesca o astillas para encender».

Asturia. Astur ombre de Asturia. VEL. DLE ${ }^{10}$.

Atramuz. Lupinus est vulgo el atramuz. DLE: «por el altramuz legumbre». Atún. Thynnus ${ }^{11}$ est vulgo el atún. VEL. DLE: «por el atún pescado».

Bala. Follis est la bala o el fuelle. VEL: «Bala de viento. follis.is.». DLE: «por la bala para jugar».

Banco de galea. Transtrum est el banco de galea. VEL. DLE: bajo la entrada Trastrum.

Barrena. Terebra est vulgo barrena. VEL. DLE.

Barro, cosa de. Testaceus est cosa de barro. DLE: «por cosa de barro cozido».

Bastardo. V. Trompeta.

Batalla. Cohors la batalla o corral. veL

Bazo. Splen vulgo est bazo. VEL: «Baço parte de la assadura. splen.enis.». DLE.

Bazo. Lyen est qui splen el bazo. VEL. DLE.

Bellota. Glans est fructus silvestris alias bellota. VEL. DLE.

Berraco. Verres est el berraco. VEL. DLE.

Birrete. Pileus est quod vulgo el birrete. DLE: «por el bonete o caperuça».

Bóveda. Fornix est arcus o bóveda. VEL. DLE: «por el arco de bóveda».

Burla. Jocus est burla de palabra. VEL. DLE.

Cadena. Torquis est collar o cadena. VEL.

Cáliz. Gaddir ciuitas hispaniae Cáliz. VEL: «Calez ciudad desta isla. Gaddir.iris.». DLE: «Gades. ium. por Cález isla de España».

Camisa. Supparus ${ }^{12}$ est que vulgo camisa. vEL: «Camisa de muger. supparus. i». DLE: «por la camisa de lino».

Canal del río. Profluens est la canal del río. DLE: «por el venaje del río».

Canasto. Quallus est el canasto. VEL. DLE: «por la canasta». Primera documentación en $I L$.

Cañahexa. Ferula est vulgo cañaheja. VEL. DLE: «por la cañaheja ierva».

Capiro. Cuculus el cuclillo o la cugulla o capiro. DLE: «por capirote o papahigo». Primera documentación en $I L$.

Carmeso. Purpura est piscis quo inficitur el carmeso. VEL: "Carmesi. purpura. ae. murex. icis.». En el DLE, con dos entradas: «por la concha del carmeso» y «por el mesmo carmesí». Primera documentación en $I L$.

\footnotetext{
${ }^{10}$ Bajo la entrada Astures.um.: «pueblos de España asturianos».

${ }^{11}$ En las Introductiones, Thymus; en el VEL, thymnus; en el DLE, thymmus.

${ }^{12}$ En las Introductiones, Suparus.
} 
Carnicol. Talus est vulgo el carnicol. VEL: «Carnicol de animal grande. talus. i.». DLE: «por el carnicol uesso del pie». Primera documentación en $I L$.

Carrizo. Carex herba est el carrizo. VEL. DLE.

Cartapacio. Albioli carthe non scriptae cartapacio. VEL: «Cartapacio. albiolus. i.». DLE: «Albiolus. i. por cartapacio en blanco».

Caspa. Furfures sunt in capite la caspa. VEL. DLE. Primera documentación en $I L$.

Caxcajo. Glarea est el caxcajo. vEL: «Cascajo arena con pedrezicas. glarea. ae.». DLE.

Celada. Insidiae asechança o celada. VEL: «Celada engaño de guerra. insidiae. arum.».

Chanela. Solea est chapín o chanela. VEL: «chinela calçado». Primera documentación en $I L$.

Chapín. Solea est chapín o chanela. VEL: «chapín de muger». DLE: «por alcorque o chapín».

Choza. Teges est vulgo choza. VEL; «Choça». DLE: «teges tegetis. por la estera».

Cicorea. Intybus herba vulgo cicorea o almirón. VEL: «Cicorea ierva o almirón. cicorium. ii.». DLE: «por la cicorea ierva».

Cobre. Aes aeris vulgo el cobre. VEL: «Cobre metal. aes cyprium». DLE: «por cobre o cualquier metal».

Cohombro. Cucumis pepino o melón o cohombro. VEL. DLE. Primera documentación en $I L$.

Colmenar. Alvearium ${ }^{13}$ vulgo colmenar. VEL: «Colmenar. alvearium.ii.». DLE.

Collar. Torquis est collar o cadena. VEL. DLE: «Torques torquis. por el collar de oro».

Comino. Cyminum vulgo el comino. DLE.

Conejo. Cuniculus el conejo o la mina. VEL. DLE.

Copa. Crater vas potorium copa. VEL: «Copa grande. crater. eris.». DLE: «Crater. eris. por gran copa».

Coral. Coralus vulgo el coral. veL. DLE.

Corazas. Thorax est corazas o cuerpo. veL: «Coraças armadura del cuerpo». DLE: «por las coraças para armar».

Corona. Corolla ${ }^{14}$ parva corona. VEL: «Corona pequeña. corolla. ae.». DLE: «Corolla.ae. por la corona pequeña».

Corral. Cohors la batalla o corral. VEL. DLE.

Corza. Dorcas ex genere cervino corza. VEL: «Corço o corça». DLE.

Coser. Sarcio est quod vulgo dicitur coser. VEL. DLE: «por coser y zurzir».

${ }^{13}$ Abuearium en las Introductiones.

${ }^{14}$ Corola en las Introductiones Latinae. 
Coxquillas. Titilo est hazer coxquillas. VEL

Cuchara. Coclear la cuchara in similitudinem cocleae. VEL. DLE. Primera documentación en $I L$.

Cuclillo. Cuculus el cuclillo o la cugulla o capiro. veL: «cuclillo ave conocida». DLE: «por el cuclillo ave». Primera documentación en $I L$.

Cuerpo. Thorax est corazas o cuerpo. VEL: «Ueco del cuerpo del animal». DLE: «por el ueco del cuerpo».

Cugulla. Cuculus el cuclillo o la cugulla o capiro. veL: «cugulla como capilla». DLE: «por la capilla o cugulla». Primera documentación en $I L$.

Dátil. Careota vulgo el dátil. vel. DLE. Primera documentación en $I L$.

Diamante. Adamas vulgo el diamante. VEL. DLE. Primera documentación en $I L$.

Donaires. Facetiae sunt los donayres. VEL. DLE: «Facetia. ae. por el donaire en palabras».

Dormidera. Papaver est la dormidera o map ${ }^{15}$. VEL. DLE: «papauer erraticum. por hamapola». Primera documentación en $I L$.

Embaymientos. Praestigie sunt los embaymientos.

Empeyne. Lichen est impetigo menti empeyne. VEL: «Empeine». DLE: «por el usagre». Primera documentación en $I L$.

Endechas. Neniae sunt quae vulgo endechas. En DLE, tiene dos entradas: «por endecha sobre muerto» y «por la niñería».

Enxundia. Adeps vulgo el enxundia. VEL. DLE.

Erizo de la castaña. Calix per i. iota el uaso; at uero per y. psilon el erizo de la castaña. VEL. DLE.

Escandia. Far est quae vulgo la escandia ${ }^{16}$. VEL: «Escandia especie de trigo. far. ador.». DLE: «Far farris. por la escanda o escandia».

Esmalte. Emblema vulgo el esmalte. VEL. DLE.

Estrado. Pulvinar est ubi sunt pulvili el estrado. VEL. DLE: «por el estrado del templo».

Faraute. Interpres est nuntius o faraute. VEL: «Faraute de lenguas». DLE. Primera documentación en $I L$.

Faze. Fascis est vulgo el faze o manojo. vEL: «Hace de cosas menudas atado. fascis. is», y «Hacecillo pequeño haze. fasciculus. i». DLE: «por el haze o carga».

Fiador. V. Abonado.

\footnotetext{
15 Puede ser hamapola.

16 En las Introductiones, scandia.
} 
Flema. Pituita est vulgo la flema. DLE: «por la flema umor».

Fornaza. Caminus latine el forno o fornaza. VEL: «hornaza», «horno». DLE: «Caminus. i. por la hornaza o horno».

Forno. Vid. Fornaza.

Francolín. Attagen vulgo el francolín. VEL. DLE.

Fuelle. Follis est la bala o el fuelle. VEL. DLE: «por el fuelle para soplar».

Galea. V. Banco de galea.

Gallego. Chorus ventus gallego. VEL. DLE.

Gama. Dama ex genere cervino la gama. VEL. DLE.

Ganado. Pecus oris est el ganado. VEL. DLE: «por todo el ganado menudo»!.

Golfín. Delphin piscis est vulgo golfín. VEL: «Golfín. lo mesmo es que delfin». DLE: «Delphin. inis. por el delfín o golfin».

Gryfo. Gryphs animal est el gryfo. VEL: «Grifo». DLE. Primera documentación en $I L$.

Guadalquivir. Bethis est fluvius in Hispanie ${ }^{17}$ Guadalquivir. VEL. DLE.

Guadiana. Anas fluvius Hispanie Guadiana. VEL. DLE: bajo la entrada Ana.ae., «por Guadiana río de España».

Harnero. Vannus ${ }^{18}$ est vulgo harnero. VEL. DLE: «por la çaranda o harnero». Primera documentación en $I L$.

Hastío. Fastidio est aver hastío. VEL. DLE: «por el mismo hastío». Primera documentación en $I L$.

Hastío. Satias est quod vulgo hastío. VEL. DLE: «Saties satiei. por el hastío».

Haya. Abies arbor est glandifera haya. DLE: «Abies. Por cierto árbol de especie de pino». VEL: «Haia árbol conocido. phagus.i.».

Hevilla. Fibula est quae vulgo la hevilla. VEL. En DLE, dos entradas: «por la hevilla de las venas» y "por el candado pequeño».

[Hierba] buena. Menta est herba quae dicunt buena. VEL: «Ierva buena». DLE.

Hígado. Jecur quod vulgo dicitur hígado. VEL. DLE. Primera documentación en $I L$.

Hoja. Frons.dis. la hoja del árbor. VEL. DLE. Primera documentación en $I L$.

Hongo. Boletus est fungus hongo. VEL. DLE.

Hoyo. Scrobs est vulgo hoyo. VEL. DLE. Primera documentación en $I L$.

Juntura. Compago vulgo la juntura. VEL: «Juntura assí. contiguitas. atis. DLE.

17 Spanie en las Introductiones.

18 En las Introductiones, [V]anus. 
Ladrillo. Laterem ladrillo o adobe. VEL. DLE.

Lagar. Lacus el lago o lagar o viga. VEL.

Lago. Lacus el lago o lagar o viga. VEL. DLE.

Lamber. Lambo est lingere lamber. VEL: «Lamer lambo.is.». DLE: «por lamber o lamer».

Láudano. Laser ${ }^{19}$ est quod dicitur láudano. VEL: «Láudano olor conocido. ladanum. i.». DLE: «Ladanum.i. por el láudano olon». Primera documentación en $I L$.

Lid. Lis est contentio el pleyto o lid. VEL. DLE.

Liga. Viscum est que vulgo la liga. VEL. DLE: «por la liga o lidia».

Lirón. Glis animal est vulgo lirón. VEL. DLE: «por el lirón animal de comer». Primera documentación en $I L$.

Majada. Magalia sunt lingua punica la majada. VEL. DLE: «por majadas del ganado».

Malva loca. Malope est malva quae vulgo loca. VEL. DLE: «por la malva loca». Primera documentación en $I L$.

Mamar. Lalo. as. est lacte ${ }^{20}$ suggere mamar. VEL. DLE: «Lallo. as. avi. por mamar leche».

Manojo. Fascis est vulgo el faze o manojo. VEL: «Manojo o manada. manipulus merges. itis». DLE: «por el haze o carga».

Marca. Picenum porcio est Italia la marca. VEL. DLE.

Marfil. Ebur dens elephantis el marfil. VEL. DLE.

Margarita. Margarites ${ }^{21}$ est quae margarita aljófar. VEL. DLE: «Margarites. ae. por el aljófar o perla».

Mastranto. Mentastrum est herba mastranto. VEL. DLE.

Melón. Cucumis pepino o melón o cohombro. VEL: «Melón fruta conocida. pepo. melopepo. onis». DLE: «Melo. onis. por el melón o papón». Primera documentación en $I L$.

Mina. Cuniculus el conejo o la mina. VEL. DLE.

Missiva. Litterae. arum. epistola missiva. VEL: «Carta mensagera. litterae. arum.». DLE: «Litterae. arum. por la letra mensajera», «Epistola. ae. por carta mensagera».

Molino. Pistrinum est el molino o aceña. VEL. DLE.

Morecillo. Musculus est el morezillo. VEL. DLE: «por el morezillo de braço». Primera documentación en $I L$.

Mot[e]. Epigramma brevis inscriptio vulgo mot[e]. VEL: «Mote o motete». DLE: «por mote en verso».

\footnotetext{
${ }^{19}$ En las Introductiones, «lasser».

${ }^{20}$ En las Introductiones, lac.

${ }^{21}$ En las Introductiones, margaris.
} 
Olla. Cacabus vulgo olla. DLE: «Cacabus. i. por la caldera para cozer».

Ordeñar. Mulgeo est quod dicunt ordeñar. VEL. DLE. Primera documentación en $I L$.

Oruga. Eruca herba vulgo la oruga. VEL. DLE: con dos entradas: una para «por la oruga ierva conocida», y otra «por el oruga gusano». Primera documentación en $I L$.

Oxear. Abigere est oxear o arredrar. VEL. Vid. arredrar. Primera documentación en $I L$.

Paje. Exoletus est qui vulgo dicitur paje. VEL: «Page». DLE: «por lo que dexó de crecer». Primera documentación en $I L$.

Papada. Glandium est in porcis la papada. VEL. DLE. Primera documentación en $I L$.

Paz. Foedus est la paz o condiciones della. VEL. DLE: «por la confederación y criança».

Pegujal. Peculium est vulgo pegujal. VEL. DLE. Primera documentación en $I L$.

Pepino. Cucumis pepino o melón o cohombro. VEL.

Pértigo. Temo est vulgo el pértigo. VEL: «Pértiga palo de cierta forma. pertica. ae.». En DLE, dos entradas: «por timón de arado» y por «pértigo de carro». Primera documentación en $I L$.

Peso grande ('balanza'). Trutina est el peso grande. VEL. DLE: «por el peso valança grande».

Piojo. Pediculus est el piojo. VEL. DLE.

Pleyto. Lis est contentio el pleyto o lid. VEL: «Pleito». DLE: «por el pleito, lid y contención».

Precio. Liceo est ser puesto en precio. DLE.

Primo. Patruelis est qui vulgo primo. VEL. DLE.

Provisión de año. Penus est vulgo la provisión de año. VEL. DLE: «por la provisión para comer».

Rayda. Rasilis cosa rayda a rado. VEL. DLE. Primera documentación en $I L$.

Reales. Castra sunt los reales de la gente. VEL: «Real de gentes armadas. castra.orum.». DLE: «Castra.orum. por el real de la ueste».

Recalcar. Farcio est impleo proprie recalcar. VEL. DLE: «por recalcar o embutir». Primera documentación en $I L$.

Red. Casses cassium est reticulum red. VEL. DLE.

Regalar. Indulgeo est dare operam o regalar. VEL. DLE. Primera documentación en $I L$.

Rehén. Obses est qui vulgo dicitur rehén. VEL. DLE.

Reja. Vomis est vulgo la reja. VEL: «Reja de hierro para arar. vomis. eris.». DLE: «Vomer vel vomis. por la reja». 
Renta. Vectigal est la renta in portatis. VEL. DLE: «por renta rentada».

Riestra. Restis est funis sive riestra. VEL: «Riestra de ajos o cebollas». DLE: «por la riestra de ajos».

Rifar de perros. Ringor proprie el rifar de perros. VEL. DLE: «por rifar como perros».

Riñón. Ren est quod vulgo riñón. DLE: «por el riñón o ren».

Rueca. Colus est vulgo la rueca. veL.

Salero. Salinum est quod dicitur salero. VEL. DLE. Primera documentación en $I L$.

Salmuera. Muria est quam vocant salmuera. VEL. DLE: «por la salmuera o salmorejo». Primera documentación en $I L$.

Saltar. Salto est saltum facere saltar. VEL. DLE.

Sevilla. Hispalis ${ }^{22}$ civitas Hispaniae Sevilla. VEL. DLE.

Sirguerito. Carduelis avicula el sirguerito. VEL. DLE.

Sobaco. Ala et axilla el sobaco. VEL. DLE: «por lo cóncavo del sobaco o pelos dél».

Soberado. Caenaculum el soberado. VEL. Primera documentación en IL.

Sueldo. Stipendium est quod vulgo sueldo. VEL. DLE: «por el sueldo o salario».

Tallo: Caulis vulgo dicitur el tallo. VEL. DLE.

Tanto. Talio est poena del tanto. VEL. DLE: «por la pena del tanto».

Tapete. Tapes est vulgo el tapete. DLE: «por alhombra o alquerifa».

Tártaro. Scytha est qui hodie tártaro. vEL.

Temblar. Tremo est quod vulgo temblar. vEL. DLE: «por temblar temiendo».

Tenazas. Forceps quae vulgo tenazas. VEL. DLE.

Tizón. Titio vulgo quod dicitur tizón. veL.

Tizón. Torris est vulgo tizón. DLE: «por el tizón o torrezno».

Topo. Talpa est que vulgo topo. VEL. DLE: «por el topo animal».

Treguas. Induciae quae vulgo dicuntur treguas. VEL. DLE.

Trinchante. Chironomon vulgo el trinchante. VEL. DLE. Primera documentación en $I L$.

Tripas. Omentum sunt las tripas. VEL. DLE: «por el redaño de las tripas».

Trompeta. Cornicen ${ }^{23}$ el trompeta bastardo. VEL: «Trompeta que tañe ésta. cornicen. inis.». DLE: «Cornicen. inis. por el trompeta bastardo».

Truhán. Scurra est qui hispane truhán. VEL. DLE: «por el truhán que hace reír».

\footnotetext{
${ }^{22}$ En las Introductiones, Hispal.

${ }^{23}$ Cornicem en las Introductiones Latinae.
} 
Ungüento. Myropola est ungüento venditor. VEL. DLE: «por el ungüentario». Untar. Lino est in unguo untar. VEL. DLE.

Vaso. Calix per i. iota el uaso; at uero per y. psilon el erizo de la castaña. VEL.

Vayo. Spadix est color in equis vayo. VEL.

Viga. Lacunar est la viga. VEL. DLE.

Viga. Lacus el lago o lagar o viga. VEL.

Vimbre. Siler est que dicitur vimbre. VEL. DLE: «por una especie de vimbre».

Xara. Myrica est quae vulgo xara. VEL. DLE: «por la xara o iniesta».

Yjadas. Ilia sunt quae vulgo las yjadas. VEL: «Ijada». DLE: «Iliacus. a. um. por doliente de la ijada».

Zarza. Rubus est quae vulgo zarza. VEL: «Çarça mata espinosa». DLE: «por la çarça mata».

Zarzo. Crates vulgo dicitur el zarzo. vEL: «Çarzo de vergas. crates. is.». DLE. 